\title{
A Microsatellite Polymorphism Associated with the PLC1 (Phospholipase C) Locus: Identification, Mapping, and Linkage to the MODY Locus on Chromosome 20
}

\author{
Cynthia B. Rothschild, * Gita Akots, * Stefan S. Fajans, † and Donald W. Bowden* \\ *Department of Biochemistry, Bowman Gray School of Medicine, Medical Center Boulevard, Winston-Salem, North Carolina 27157; \\ and TDepartment of Internal Medicine, University of Michigan Medical Center, Ann Arbor, Michigan 48109 \\ Received September 25, 1991; revised February 25, 1992
}

\begin{abstract}
A highly polymorphic $(\mathrm{dC}-\mathrm{dA})_{n} \cdot(\mathrm{dG}-\mathrm{dT})_{n}$ dinucleotide repeat at the PLC1 locus on human chromosome 20 has been identified. Primers flanking the dinucleotide repeat were used for PCR amplification of the repeat region in 37 informative kindreds from the Centre d'Etude du Polymorphisme Humain. Two-point linkage analysis indicates that PLC1 is closely linked to several chromosome 20 markers, including D20S16 $\left(z_{\max }\right.$ $=41.25 ; \hat{\theta}=0.07), \operatorname{D20S17}\left(z_{\max }=42.81 ; \hat{\theta}=0.09\right)$, and ADA $\left(z_{\max }=57.24 ; \hat{\theta}=0.05\right)$. Multipoint linkage analysis places the PLC1 locus between D20S18 and D20S17, 11.2 and $6.6 \mathrm{cM}$, respectively, from these loci (sex-averaged distances). In addition, the PLC1 gene shows linkage to the maturity-onset diabetes of the young (MODY) locus on chromosome 20 with a lod score of 4.57 at $\hat{\theta}=0.089$. 1992 Academic Press, Inc.
\end{abstract}

\section{INTRODUCTION}

Recently, a locus responsible for maturity-onset diabetes of the young (MODY), a form of non-insulin-dependent diabetes mellitus (NIDDM), has been mapped to chromosome 20 in a large, multigeneration pedigree called the R.-W. pedigree. Bell et al. (1991) reported linkage between MODY and the adenosine deaminase (ADA) gene with a lod score of 5.25 at a recombination fraction of 0.00 . In our laboratory, multipoint linkage analysis with nine RFLP loci maps the MODY locus to an approximately $20-\mathrm{cM}$ region including D20S16 and D20S14/D20S18 (Bowden et al., 1992a,b). There is significant evidence for genetic heterogeneity in MODY, but branches of the R. $-W$. family with clear early onset of diabetes have a lod score of greater than 10 at $\theta=$ 0.001 with marker D20S16 (Bowden et al., 1992b). We are therefore interested in mapping additional markers in this region of the chromosome, particularly genes that might be candidates for the MODY locus.

One such candidate is the PLC1 gene, which codes for phospholipase C. The intracellular second messengers diacylglycerol and inositol 1,4,5-triphosphate are known to be important in the regulation of cellular activity (re- viewed in Berridge, 1987), and their production in response to the binding of specific agonists to cellular receptors is mediated by activated phosphatidylinositolspecific phospholipase C (PLC) enzymes. There is good evidence for a primary role of PLC, phosphoinositide metabolism, and activation of $\mathrm{C}$ kinases in the secretory processes that regulate glucose-stimulated insulin secretion (Zawalich, 1988). In addition, phospholipase $\mathrm{C}$ mimics the action of insulin on adipose tissue (Honeyman et al., 1983). This suggests that PLC could play a role in MODY. Stahl et al. (1988) have reported the cloning and sequencing of a cDNA (PLC-148) encoding a bovine phosphatidylinositol-specific PLC. Interestingly, PLC148 displays striking sequence similarity with the noncatalytic domain of the nonreceptor tyrosine kinases (e.g., src; Stahl et al., 1988). PLC-148 was subsequently used to isolate a human genomic clone that by in situ hybridization mapped the human gene (PLC1) to chromosome 20 at a position of q12-q13.1 (Bristol et al., 1988). Dinucleotide repeat sequences of the form (dC$\mathrm{dA})_{n} \cdot(\mathrm{dG}-\mathrm{dT})_{n}$ are abundant repetitive DNA elements that exhibit length polymorphisms (Litt and Luty, 1989; Weber and May, 1989). We have characterized such a dinucleotide repeat polymorphism at the PLC1 locus, and linkage analysis was used to map the PLC1 gene with respect to the MODY locus and other genetic markers on chromosome 20.

\section{MATERIALS AND METHODS}

The PLC-148 cDNA subcloned in Bluescript (Stahl et al., 1988) was obtained from J. Knopf (Genetics Institute, Cambridge, MA). This clone did not reveal any restriction fragment length polymorphisms (RFLPs) when five individuals were tested with a variety of restriction enzymes (BamHI, BglII, EcoRI, HincII, HindIII, MspI, PstI, $R s a \mathrm{II}$, and $T a q \mathrm{I})$. Therefore, overlapping cosmid clones were isolated using a previously described screening method (Bowden et al., 1988) and were subsequently assayed for the presence of $(\mathrm{dC}-\mathrm{dA})_{n} \cdot(\mathrm{dG}$ $\mathrm{dT})_{n}$ dinucleotide repeats by hybridization of Southern blots (as described in Donis-Keller et al., 1987) of EcoRI-digested cosmids with radiolabeled $(\mathrm{dC}-\mathrm{dA})_{n} \cdot(\mathrm{dG}-\mathrm{dT})_{n}$ polynucleotide (Pharmacia). The blots were washed using a modification of the conditions described by Weber and May (1989), for $15 \mathrm{~min}$ at $25^{\circ} \mathrm{C}$ in $2 \times \mathrm{SSC}$, followed by 30 min at $65^{\circ} \mathrm{C}$ in $2 \times \mathrm{SSC}, 25 \mathrm{mM} \mathrm{Na}{ }_{2} \mathrm{PO}_{4}(\mathrm{pH} 6), 0.1 \% \mathrm{SDS}$, and $30 \mathrm{~min}$ 
at $65^{\circ} \mathrm{C}$ in $1 \times \mathrm{SSC}, 0.1 \%$ SDS. One cosmid, PLC/S104, contained a fragment of $>25 \mathrm{~kb}$ that hybridized to the $(\mathrm{dC}-\mathrm{dA})_{n} \cdot(\mathrm{dG}-\mathrm{dT})_{n}$ polynucleotide. This fragment was incubated with Sau $3 \mathrm{~A}$ restriction endonuclease, and the products were cloned into M13mp18. Nitrocellulose filter plaque lifts from the resulting recombinants were again screened by hybridization to ${ }^{32} \mathrm{P}$-labeled $(\mathrm{dC}-\mathrm{dA})_{n} \cdot(\mathrm{dG}-\mathrm{dT})_{n}$ polynucleotide, and dinucleotide repeat regions were identified by DNA sequencing.

Primers designed from the DNA sequence flanking the dinucleotide repeat region were analyzed using the Genetics Computer Group Inc. (Madison, WI) sequence analysis software (Devereux et al., 1984). Primer sequences showing significant potential secondary structure or homology to repetitive DNA elements (e.g., Alu repeats) were not used. The PLC1 primers PLCpr1 5'-AACCAGTCTGCTCTTCCGGTG-3' and PLCpr2 5'-CTGCCT'TCAACTGATCTCAATGG-3' were synthesized by Operon Technologies Inc. (Alameda, CA). Polymerase chain reactions were carried out in a $25-\mu 1$ volume essentially as described by Weber and May (1989), with the exception that $1 \mathrm{mM}$ spermidine was included in all samples. Samples were processed through 35 temperature cycles consisting of $1 \mathrm{~min}$ at $94^{\circ} \mathrm{C}, 1.5 \mathrm{~min}$ at $58^{\circ} \mathrm{C}$, and $2 \mathrm{~min}$ at $72^{\circ} \mathrm{C}$, with the last elongation step lengthened to 7 min, and the products were analyzed on standard sequencing gels $(6 \%)$ as described in Weber and May (1989).

The PIC1 dinucleotide repeat polymorphism was genotyped on 37 families from the Centre d'Etude du Polymorphisme Humain (CEPH). Genotypic data for other probes were derived from the CEPH Version 4 database. Linkage analysis in the CEPH families was carried out using the CRI-MAP program (Version 2.4) (DonisKeller et al., 1987), which can rapidly perform multipoint linkage calculations simultaneously with large numbers of loci. The TWOPOINT option of CRI-MAP was used for sex-equal and sex-specific two-point linkage calculations with PLC1. PLC1 placement on chromosome 20 using multipoint analysis was calculated using the BUILD option of CRI-MAP, which constructs a map(s) from an unordered set of loci, and the ALL option, which placed PLC1 in a preexisting order of loci. Both approaches gave identical locations. Corresponding maximum likelihood recombination fractions (both sex-equal and sex-specific) and map distances (using Kosambi mapping function) were determined using the FIXED option. The CHROMPICS option of CRI-MAP was used to rigorously check genotypic data. Any PLC1 genotypings leading to the appearance of isolated double crossovers were repeated.

Two-point linkage analysis of PLC1 and the MODY locus was carried out using the ILINK and MLINK options of the LINKAGE program (Version 4.9) since this program allows calculations to be based upon age-dependent penetrance (Lathrop et al., 1984). A stepwise age-at-onset estimate assuming complete penetrance was used. Liability classes and other parameters are discussed in detail in Bowden et al. (1992b). The LINKMAP option of LINKAGE was used for multipoint analysis of MODY genotypic data; this program calculates lod scores of one locus against a fixed map of other loci. Lod scores reported are from analysis of the two branches of the R.-W. family (branches II,2 and II,5) which are clearly linked to chromosome 20 and have NIDDM onset at less than 25 years of age (see Bowden et al., 1992b).

\section{RESULTS}

\section{Identification of the PLC1 Dinucleotide Repeat Polymorphism and Linkage Mapping of PLC1}

When several conventional restriction endonucleases (as described under Materials and Methods) were used, the PLC-148 cDNA clone did not reveal restriction fragment length polymorphisms. Two overlapping cosmids that hybridized to PLC-148 cDNA were isolated from a genomic library and screened for the presence of dinucleotide repeats by hybridization with ${ }^{32} \mathrm{P}$-labeled (dC$\mathrm{dA})_{n} \cdot(\mathrm{dG}-\mathrm{dT})_{n}$ polynucleotides. Upon subcloning and

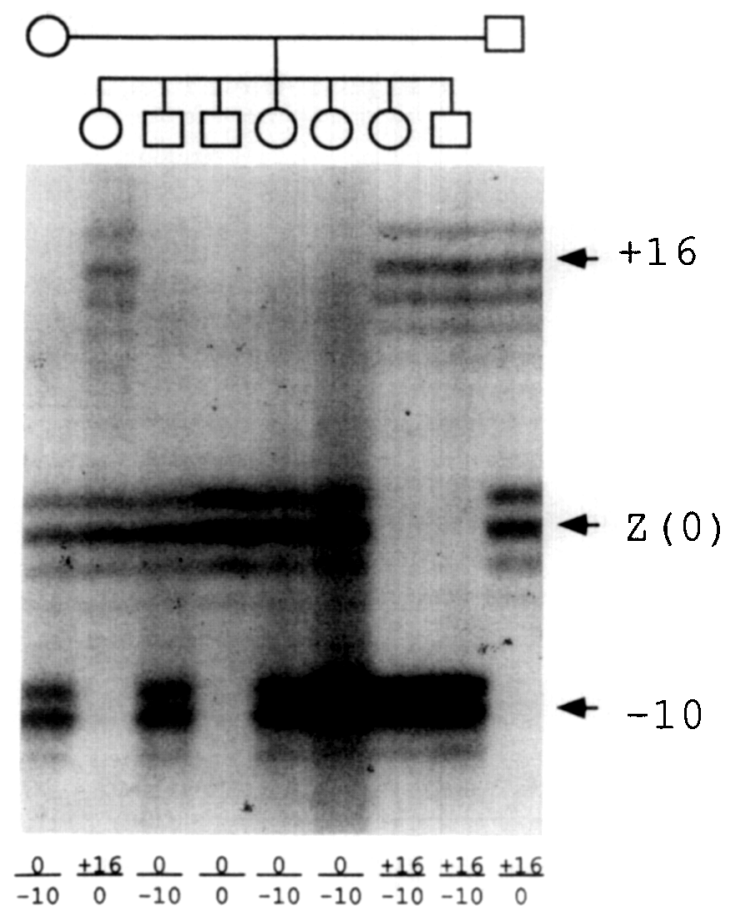

FIG. 1. Gentoyping of the PLC1 dinucleotide repeat on CEPH family 28 . Individuals from CEPH family 28 and their corresponding alleles from PCR amplification of the PLC1 dinucleotide repeat are shown above and below the autoradiogram, respectively. The position of each allele is indicated by the arrows. Allele sizes are $Z(0)=166$ nucleotides; $+16=182$ nucleotides; $-10=156$ nucleotides.

DNA sequence analysis, the cosmid PLC/S104 was found to have a perfect dinucleotide repeat sequence of 42 nucleotides in length (i.e., 21 repeats). Primers flanking the PLC1 dinucleotide repeat were used for PCR amplification of this region with parent DNAs from 40 CEPH kindreds. The PLC1 dinucleotide repeat was informative in 37 of 40 families (59 of 78 individuals were heterozygous), with a corresponding PIC of 0.72 . The PLC1 dinucleotide repeat polymorphism was then genotyped on the 37 informative families, which amounted to 431 informative meioses. An example of the segregation of the polymorphism in CEPH kindred 28 is shown in Fig. 1. As is typical of PCR amplification of dinucleotide repeats, multiple bands are seen for each allele. Thus, the (lighter) band above the indicated allele most likely corresponds to the slower moving GT-containing strand, whereas the other minor bands are likely due to slippage during PCR (discussed in Weber and May, 1989; Litt and Luty, 1989). In practice, the additional minor bands present little problem in accurate genotyping. The varying allele sizes and their frequencies for 109 unrelated individuals in the CEPH pedigrees are given in Table 1. Except for the $Z(0)$ allele, with a frequency of $46.8 \%$, the other 13 alleles were well distributed over a range of frequencies $(0.9-10.6 \%)$, making the polymorphism highly informative.

The data from the CEPH PLC1 genotyping were merged with the CEPH (Version 4) genotypic data. Also included in the analysis were the ADA AluVpA polymorphism (Economou et al., 1990; data kindly provided 
TABLE 1

PLC Dinucleotide Repeat Allele Frequency

\begin{tabular}{cc}
\hline Allele & Frequency \\
\hline+18 & 0.014 \\
+16 & 0.023 \\
+14 & 0.018 \\
+12 & 0.023 \\
+10 & 0.087 \\
+8 & 0.106 \\
+6 & 0.087 \\
+4 & 0.041 \\
+2 & 0.028 \\
$\mathrm{Z}(0)$ & 0.468 \\
-2 & Not observed \\
-4 & 0.005 \\
-6 & 0.032 \\
-8 & Not observed \\
-10 & 0.060 \\
-12 & Not observed \\
-14 & Not observed \\
-16 & 0.009 \\
\hline
\end{tabular}

Note. Frequency of allele sizes generated by PCR amplification us ing PLC primers with 109 unrelated individuals in the CEPH pedigrees; $Z(0)$ allele $=166$ nucleotides.

by S. E. Antonarakis) and a dinucleotide repeat polymorphism (PIC $=0.53$ ) from the D20S17 locus (C. Rothschild, unpublished results). The results of twopoint linkage analysis are shown in Table 2. A lod table for sex-equal analysis of chromosome 20 markers with $z_{\max }>3.0$ is shown. In addition, the $z_{\max }, \hat{\theta}$ for sex-specific analysis, and the corresponding $\chi^{2}$ evaluation (Ott, 1985) of the difference between the male and female recombination fractions are shown. PLC1 displays tight linkage with D20S16 $\left(z_{\max }=41.25\right), \mathrm{D} 20 \mathrm{~S} 17\left(z_{\max }=\right.$ $42.81)$, and the ADA locus $\left(z_{\max }=57.24\right)$. For all markers except D20S18, the maximum likelihood estimates of the recombination fractions for females are significantly $(P<0.05)$ greater than those for males.

Multipoint analysis places PLC1 between D20S17 and D20S18, approximately $6.6 \mathrm{cM}$ from D20S17 and $11.2 \mathrm{cM}$ from D20S18. In Fig. 2, the region of the maximum likelihood map spanning D20S5 to D20S4 is shown. The odds against alternative placements for PLC1 are also shown; PLC1 is $>10^{4}: 1$ more likely to be in the interval flanked by D20S17 and D20S18 than in any other interval. This placement of PLC1 is in agreement with previous in situ mapping studies that localized PLC1 to q12-q13.1 (Bristol et al., 1988) and D20S16 to y13.1 (N. Rao, personal communication). When the $\chi^{2}$ test for heterogeneity was applied to the sex-equal and sex-specific multipoint $\log$ likelihoods for the region of the map from D20S5 to D20S4 (seven markers), it was found that the recombination rates for this region of the chromosome are significantly higher $\left(P<2.5 \times 10^{-6} ; 6\right.$ degrees of freedom) for females than for males. Thus, multipoint linkage analysis mapped PLC1 $21.7 \mathrm{cM}$ from D20S18 and $12.0 \mathrm{cM}$ from D20S17 using female meioses, but only $5.0 \mathrm{cM}$ from D20S18 and $2.0 \mathrm{cM}$ from D20S17 using male meioses.

\section{Linkage of the PLC1 Locus to MODY}

The PLC1 dinucleotide repeat polymorphism was genotyped on the R. $\mathrm{W}$. family, a large pedigree in which linkage of chromosome 20 markers to MODY was established (Bell et al., 1991; Bowden et al., 1992a). Two branches of the family (II,2 and II,5) have clinically documented MODY, i.e., NIDDM onset at less than 25 years of age, while there is no evidence of early onset in the other two branches (II,3 and II,6; Bowden et al., 1992b). Using the LINKAGE program (Version 4.9) (Lathrop et al., 1984), the PLC1 dinucleotide repeat shows linkage to MODY in the II,2 and II, 5 branches of the R.-W. family, with a maximum lod score of 4.57 and a maximum likelihood estimate of the recombination fraction of $\hat{\theta}=0.089$, with a $95 \%$ confidence interval of $0.025 \leqslant \theta \leqslant 0.235$. There are five obligate recombinants (individuals III-32, III-35, IV-138, IV-164, and IV-168; Bowden et al., 1992a), thus excluding PLC1 as a candidate gene for MODY. Analysis of the II,3 and II,6 (late onset) branches of the R.-W. family showed no evidence for linkage (i.e., $z<0$ for $\theta<0.5$ ).

Results of multipoint linkage analysis of MODY with loci spanning D20S4 to D20S5 are summarized in Fig. 2. The $95 \%$ confidence interval for MODY (maximum

TABLE 2

Two-Point Linkage Analysis: LODS $>3$

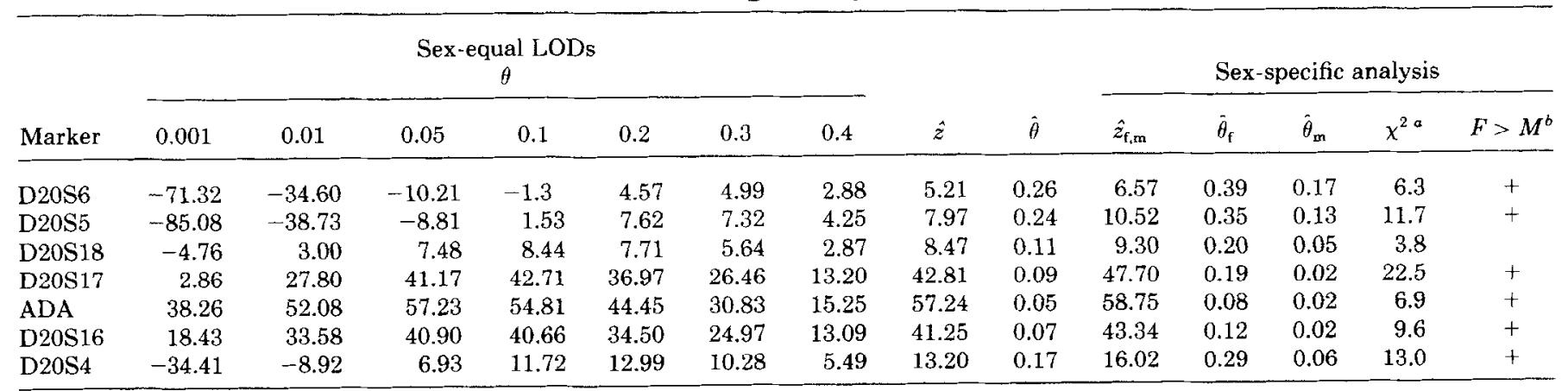

${ }^{a} \chi^{2}>3.841$ corresponds to $P<0.05$ (one degree of freedom); $\chi^{2}>7.879$ corresponds to $P<0.005 ; \chi^{2}>10.828$ corresponds to $P<0.001$.

${ }^{b} F>M$ : Female length significantly greater than male length. 

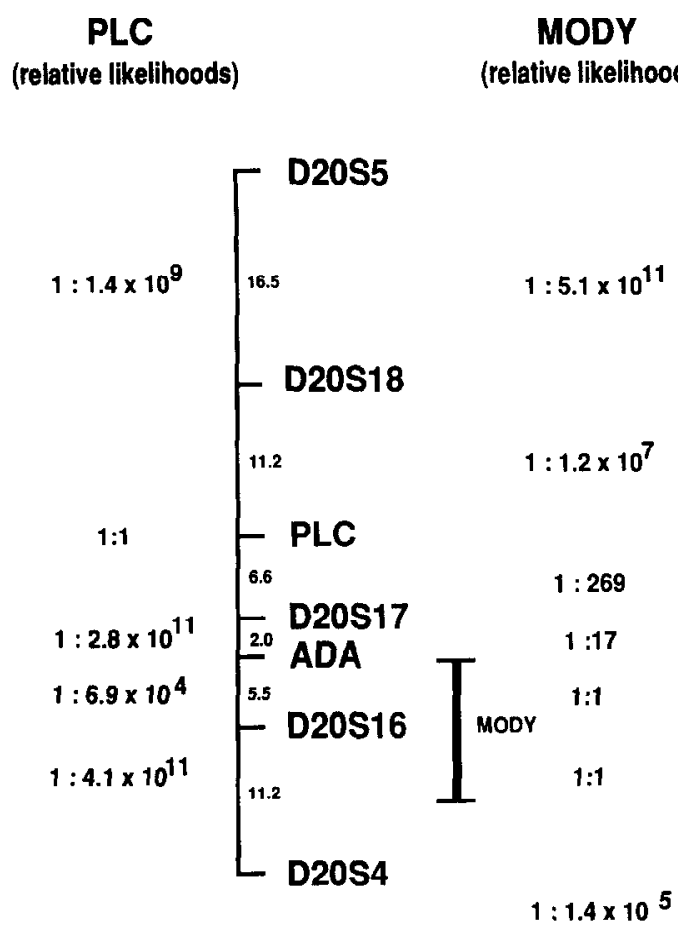

FIG. 2. Multipoint mapping of the PLC1 and MODY genes on chromosome 20. PLC1 placement on chromosome 20 was calculated using both the BUILD and ALL options of CRI-MAP. The corresponding genetic distances (sex-equal) are scaled in centimorgans and were derived (Kosambi mapping function) from the maximum likelihood recombination fractions using the FIXED option of CRI-MAP. Only the region of chromosome 20 spanning D20S5 to D20S4 is shown. Odds of alternative placements for PLC1, calculated using the ALL option of CRI-MAP, are shown to the left of the map (all other placements of PLC1 along the chromosome are $>10^{20}: 1$ less likely). MODY location on the chromosome 20 map was calculated using the LINKMAP option of LINKAGE. The most likely placement for MODY was defined as the area under the LOD curve including maximum LOD - 1 . Odds for placement of MODY in each interval were calculated using the maximum LOD for that interval and are shown at right.

$\mathrm{LOD}=12.67)$ is an $11-\mathrm{cM}$ region centered at D20S16. The odds against placement of MODY in each interval along the map are also shown.

\section{DISCUSSION}

Through locus expansion by cosmid walking, we have isolated a genomic clone for the PLC1 gene. The genomic clone contained a polymorphic dinucleotide repeat sequence of the form $(\mathrm{dC}-\mathrm{dA})_{21}$ with a PIC $=0.72$. The high PIC value agrees well with the values reported in the analysis by Weber (1990), where in general, it was found that the informativeness of $(\mathrm{dC}-\mathrm{dA})_{n} \cdot(\mathrm{dG}-\mathrm{dT})_{n}$ polymorphisms increases with an increasing number of repeats to a maximum PIC of approximately 0.8 at $21-24$ (dC-dA) repeats. In parallel surveys we have found that $>60 \%$ of cosmids contain dinucleotide repeats of the $(\mathrm{dC}-\mathrm{dA})_{n} \cdot(\mathrm{dG}-\mathrm{dT})_{n}$ type, as might be predicted if the approximately 50,000 such repeats (Weber and May, 1989) are randomly dispersed throughout the genome.

Multipoint analysis places PLC1 between D20S18 and D20S17, with two-point lod scores of $>40$ for PLC and the highly informative markers at D20S17, ADA, and D20S16. $\chi^{2}$ analysis of both the two-point and the multipoint data indicated that except for the interval between PLC1 and D20S18, there is a significant $(P<$ 0.05 ) increase in female vs male recombination rates for the region spanning D20S5 to D20S4. An increase in female recombination rates for this region of chromosome 20 is consistent with the microsatellite map of Hazan et al. (1992) and has been documented in earlier maps of the chromosome (Donis-Keller et al., 1987; Nakamura et al., 1989), although the reported values for genetic lengths vary. Thus, for the interval D20S4 to D20S5, multipoint analysis with PLC1 results in female and male genetic lengths of 87 and $27 \mathrm{cM}$, respectively, compared to previously reported lengths of 137 and 22 cM (Donis-Keller et al., 1987) and 35 and $18 \mathrm{cM}$ (Nakamura et al., 1989), respectively. These earlier maps, however, had few markers in this region of the chromosome. It would be expected that the addition of highly informative markers (e.g., ADA and the PLC1 and D20S17 dinucleotide repeats) should give an increasingly accurate estimate of genetic length.

The PLC1 locus shows significant linkage to MODY $\left(z_{\max }=4.57 ; \hat{\theta}=0.089\right)$. Multipoint linkage analysis strongly suggests (with odds $>10^{5}: 1$ ) that the MODY locus lies within the region defined by PLC1 and D20S4. The most likely placement for MODY is within an 11cM region centered at D20S16. Although essentially in agreement with our earlier analysis (Bowden et al., 1992a,b), inclusion of the PLC1 data has narrowed the LOD curve and shifted the most likely placement for MODY away from D20S17 and toward D20S16. The observation of multiple obligate crossovers eliminates PLC1 as a candidate gene for MODY, but the PLC1 dinucleotide repeat polymorphism should be useful in establishing linkage (or lack thereof) between the chromosome 20 MODY locus and diabetes in kindreds other than the R.-W. family.

Evidence suggests there are a number of distinct enzymes with phosphatidylinositol-specific PLC activity (Ryu et al., 1987; Hoffman and Majerus, 1982). In light of the importance of these enzymes in the regulation of cellular activity, as well as the similarity between PLC148 and known oncogenes, the polymorphism described here may allow evaluation of a role for the PLC1 gene in oncogenesis. In addition, the PLC1 polymorphism may help in delimiting the deletions on chromosome 20 seen in some myelodysplastic syndromes such as polycythemia vera (e.g., Rege-Cambrin et al., 1987).

\section{ACKNOWLEDGMENTS}

This work was supported by NIH Grant R01-DK41269 and a grant from the North Carolina Biotechnology Center. We thank Kathy Falls of Collaborative Research, Inc., for her help in setting up the LINKAGE analysis program.

\section{REFERENCES}

Bell, G. I., Xiang, K.-S., Newman, M. V., Wu, S.-H., Wright, L. G., Fajans, S. S., Spielman, R. S., and Cox, N. J. (1991). Gene for non- 
insulin-dependent diabetes mellitus (maturity-onset diabetes of the young subtype) is linked to DNA polymorphism on human chromosome 20q. Proc. Natl. Acad. Sci. USA 88: 1484-1488.

Berridge, M. J. (1987). Inositol triphosphate and diacylglycerol: Two interacting second messengers. Annu. Rev. Biochem. 56: 159-193.

Bowden, D. W., Müller-Kahle, H., Fulton, T. R., Gravius, T. C., Barker, D. F., and Donis-Keller, H. (1988). Studies on locus expansion, library representation, and chromosome walking using an efficient method to screen cosmid libraries. Gene 71: 391-400.

Bowden, D. W., Gravius, T. C., Green, P., Falls, K., Wurster-Hill, D., Noll, W., Müller-Kahle, H., and Donis-Keller, H. (1989). A genetic linkage map of 32 loci on human chromosome 10. Genomics 5: 718726.

Bowden, D. W., Gravius, T. C., Akots, G., and Fajans, S. (1992a). Linkage analysis of maturity onset diabetes of the young to RFLP loci on human chromosome 20. Diabetes 41: 88-92.

Bowden, D. W., Akots, G., Rothschild, C. B., Falls, K. F., Sheehy, M. J., Hayward, C., Mackie, A., Baird, J., Brock, D., Antonarakis, S. E., and Fajans, S. (1992b). Linkage analysis of maturity onset diabetes of the young (MODY): Genetic heterogeneity and nonpenetrance. Am. J. Hum. Genet. 50: 607-618.

Bristol, A., Hall, S. M., Kriz, R. W., Stahl, M. L., Fan, Y. S., Byers, M. G., Eddy, R. L., Shows, T. B., and Knopf, J. L. (1988). Phospholipase C-148: Chromosomal location and deletion mapping of functional domains. Cold Spring Harbor Symp. Quant. Biol. 53: 915920 .

Devereux, J., Haeberli, P., and Smithies, O. (1984). A comprehensive set of sequence analysis programs for the VAX. Nucleic Acids Res. 12: $387-395$

Donis-Keller, H., Green, P., Helms, C., Cartinhour, S., Weiffenbach, B., Stephens, K., Keith, T. P., Bowden, D. W., Smith, D. R., Lander, E. S., Botstein, D., Akots, G., Rediker, K. S., Gravius, T., Brown, V. A., Rising, M. B., Parker, C., Powers, J. A., Watt, D. E., Kauffman, E. R., Bricker, A., Phipps, P., Müller-Kahle, H., Fulton, T. R., Ng, S., Schumm, J. W., Braman, J. C., Knowlton, R. G., Barker, D. F., Crooks, S. M., Lincoln, S. E., Daly, M. J., and Abrahamson, J. (1987). A genetic linkage map of the human genome. Cell 51:319337.

Economou, E. P., Bergen, A. W., Warren, A. C., and Antonarakis, S. E. (1990). The polydeoxyadenylate tract of Alu repetitive elements is polymorphic in the human genome. Proc. Natl. Acad. Sci. USA 87: 2951-2954.
Hazan, J., Dubay, C., Pankowiak, M.-P., Becuwe, N., and Weissenbach, J. (1992). A genetic linkage map of human chromosome 20 composed entirely of microsatellite markers. Genomics 12: 183189.

Hoffman, S. L., and Majerus, P. W. (1982). Identification and properties of two distinct phosphatidylinositol-specific phospholipase C enzymes from sheep seminal vesicular glands. J. Biol. Chem. 257: 6461-6469.

Honeyman, T. W., Strohsnitter, W., Scheid, C. R., and Schimmel, R. (1983). Phosphatidic acid and phosphatidylinositol labeling in adipose tissue. Biochem. J. 212: 489-498.

Lathrop, G. M., Lalouel, J.-M., Julier, C., and Ott, J. (1984). Strategies for multi-locus linkage analysis in humans. Proc. Natl. Acad. Sci. USA 81: 3443-3446.

Litt, M., and Luty, J. A. (1989). A hypervariable microsatellite revealed by in vitro amplification of a dinucleotide repeat within the cardiac muscle actin gene. Am. J. Hum. Genet. 44: 397-401.

Nakamura, Y., Leppert, M., O'Connell, P., Lathrop, M., Lalouel, J.-M., and White, R. (1989). A genetic linkage map of markers for human chromosome 20. Genomics 5: 945-947.

Ott, J. (1985). "Analysis of Human Genetic Linkage," Johns Hopkins Univ. Press, Baltimore.

Rege-Cambrin, G., Mecucci, M., Tricot, G., Michaux, J.-L., Louwagie, A., Van Hove, W., Francart, H., and Van den Berghe, H. (1987). A chromosomal profile of polycytemia vera. Cancer Genet. Cytogenet. 25: $233-245$.

Ryu, S. H., Suh, P., Cho, K. S., Lee, K. Y., and Rhee, S. G. (1987). Bovine brain cytosol contains three immunologically distinct forms of inositolphospholipid-specific phospholipase C. Proc. Natl. Acad. Sci. USA 84: 6649-6653.

Stahl, M. L., Ferenz, C. R., Kelleher, K. L., Kriz, R. W., and Knopf, J. L. (1988). Sequence similarity of phospholipase $\mathrm{C}$ with the noncatalytic region of src. Nature 332: 269-272.

Weber, J. L., and May, P. E. (1989). Abundant class of human DNA polymorphisms which can be typed using the polymerase chain reaction. Am. J. Hum. Genet. 44: 388-396.

Weber, J. L. (1990). Informativeness of human $(\mathrm{dC}-\mathrm{dA})_{n} \cdot(\mathrm{dG}-\mathrm{dT})_{n}$ polymorphisms. Genomics 7: 524-530.

Zawalich, W. S. (1988). Modulation of insulin secretion from B-cells by phosphoinositide-dcrived second-messenger molecules. Diabetes 37: 137-141. 